\title{
Black point compensation and its influence on image appearance
}

\author{
Authors: Dragoljub Novaković ${ }^{1}$, Igor Karlović ${ }^{1}$, Ivana Tomić ${ }^{1}$ \\ ${ }^{1}$ Faculty of Technical Sciences, Graphic Engineering and Design, Novi Sad, Serbia
}

\begin{abstract}
Black point compensation (BPC) is a feature developed by the Adobe company in order to address the problem caused by differences between the darkest level of black achievable on input and output devices and it's not a part of ICC specifications. If it's turned on - black colour of input device will be mapped into the black colour of an output device; if it's not - mapping will be performed regarding the rendering intents. By default this option is turned on, which is a good solution in most cases. The aim of the work is to accurately define when black point compensation should be activated, and when not, and also to investigate influence of different colour management modules on colours allocation after conversions, in accordance to their psychophysical values. Regarding theoretical background and results of experimental part of this work conclusions about BPC usability are carried out.
\end{abstract}

Key words: rendering intent, mapping, colour appearance

\section{Introduction}

Precise definining reproduction characteristics of all devices in print production chain, as well as defining conversions between input and output devices is one of the main aim of colour management system. In profiles, basic elements of open colour management system, is well defined how white colour of an input device is converted into the white colour of output device. The exact form of converting these values, like all the other chromatic values, is specified in rendering intents. Mapping of a black colour, nevertheless, is not specially defined (Adobe Systems Incorporated, 2006).

\section{Theoretical part}

Black point compensation is used to preserve overall colours ratio on devices with different gamuts. Without compensation the darkest tones would be lost if the input device is capable of reproducing higher density

First received: 15.07.2010.

Accepted: 10.09.2010. values then output device (Figure 1). If the destination colour space has smaller gamut then source colour space, enabling BPC while mapping the darkest values will preserve dynamic range of a device, but with certain compression (Nate, 2004). That is why BPC is often considered as a gamut compression algorithm (Bonnier et al., 2008).

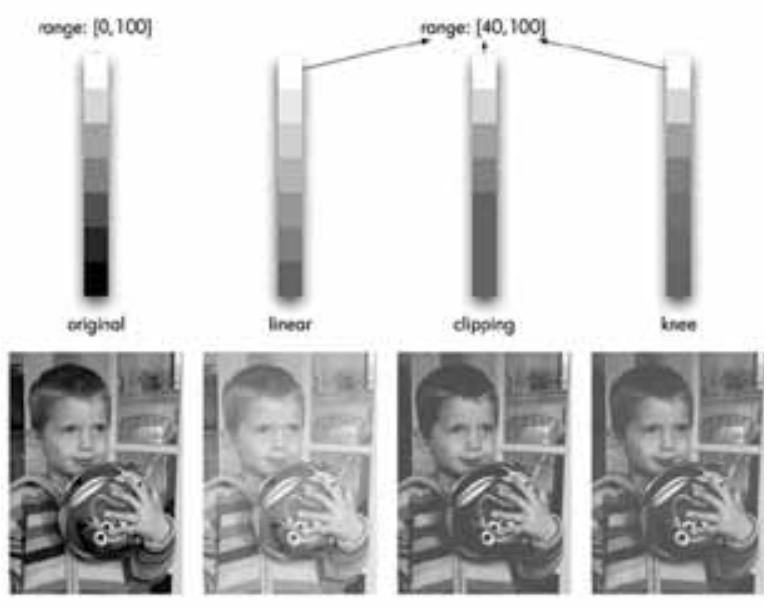

Figure 1. Example of tone mapping during conversion from larger to smaller gamut colour space 
Black point compensation should not be activated if conversion is made from smaller to larger gamut colour space (CMYK-RGB, for instance). In this case, mapping both white and black will cause incorrect representation of source colours, which can be a significant problem if precise reproduction is an aim. Example of this type of conversion is calculation from output device colour space to display colour space in soft proofing, where using BPC is generally not recommended. Black point compensation precedes other gamut mapping operations and is based on linear scaling in XYZ colour space. Source's XYZ colour space is scaled to that of the destination, where difference in XYZ values for black colour is defined as follows (Morovič, 2008):

$$
\left[\begin{array}{l}
X b p c \\
Y b p c \\
Z b p c
\end{array}\right]=\left[\begin{array}{c}
X w \\
Y w \\
Z w
\end{array}\right]-\left[\begin{array}{ccc}
\frac{Y w-Y b p d}{Y w-Y b p s} & 0 & 0 \\
0 & \frac{Y w-Y b p d}{Y w-Y b p s} & 0 \\
0 & 0 & \frac{Y w-Y b p d}{Y w-Y b p s}
\end{array}\right]\left[\begin{array}{c}
X w-X s \\
Y w-Y s \\
Z w-Z s
\end{array}\right](1.1)
$$

Where elements are defined as:

$\mathrm{X}_{\mathrm{bpc}}, \mathrm{Y}_{\mathrm{bpc}} \mathrm{i} \mathrm{Z}_{\mathrm{bpc}}$ - compensation magnitude

$\mathrm{X}_{\mathrm{w}}, \mathrm{Y}_{\mathrm{w}}$ i $\mathrm{Z}_{\mathrm{w}}$ - white point in PCS (usually D50)

$\mathrm{X}_{\mathrm{bps}}, \mathrm{Y}_{\mathrm{bps}}, \mathrm{Z}_{\mathrm{bps}}$ - black point in source colour space

$\mathrm{X}_{\mathrm{bpd}}, \mathrm{Y}_{\mathrm{bpd}}, \mathrm{Z}_{\mathrm{bpd}}$ - black point in destination colour space

$\mathrm{X}_{\mathrm{s}}, \mathrm{Y}_{\mathrm{s}}, \mathrm{Z}_{\mathrm{s}}-$ white point in source colour space.

If the lowest luminance value of pixel in an image is taken into consideration, compensation can be defined as follows (Bonnier et al., 2008):

$Y^{i}$ lowBPC $=\frac{\text { Yilow }- \text { Yminlow }}{1-\text { Yminlow }}(1-$ YminDest $)+$ YminDest

Where elements are:
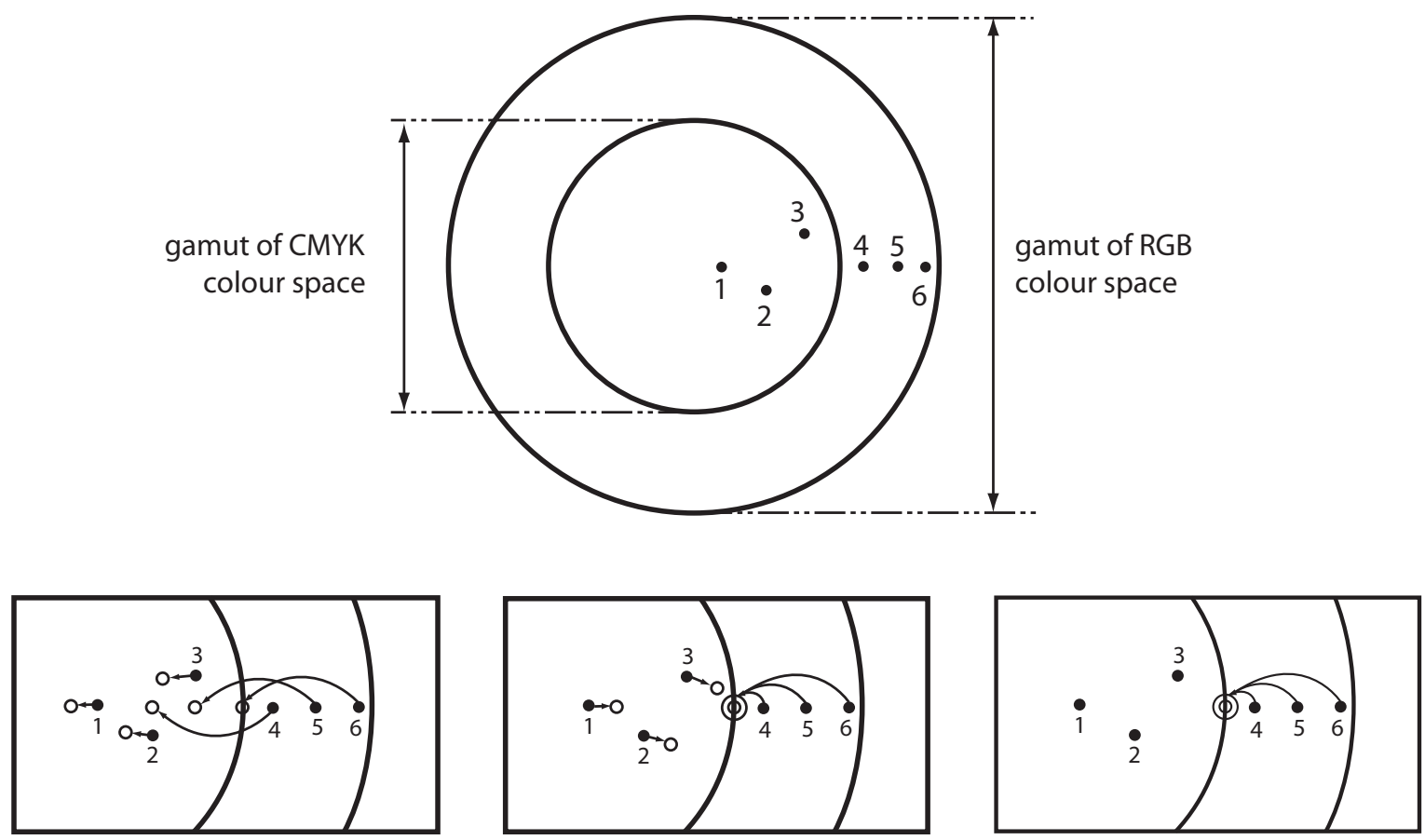

Figure 2. Illustration of mapping methods when converting colours from RGB to CMYK colour space (dots marked with 1, 2 and 3 are within gamuts of both spaces, while 4, 5 and 6 are outside destination space gamut)
$\mathrm{Y}_{\text {lowBPC }}^{\mathrm{i}}$ - scaled $\mathrm{Y}$ value of the destination pixel $i$,

$\mathrm{Y}_{\text {low }}^{\mathrm{i}}$ - the $\mathrm{Y}$ value of the source pixel $i$,

$\mathrm{Y}_{\text {minlow }}$ - the minimum $\mathrm{Y}$ value of the image, device.

By minimum Y value of the destination space lowest luminance value in that space is taken into consideration or in specific workflow lowest black value that can be obtained on output device. The same ratio stand when calculating $\mathrm{X}_{\text {lowBPC }}^{\mathrm{i}}$ and $\mathrm{Z}_{\text {lowBPC}}^{\mathrm{i}}$.

BPC option is just an additional element to be considered during colour conversions, while rendering intents precisely define how to transform out-of-gamut colours. Depending of effect which is to be attained, one of four rendering intents, defined by ICC specifications, can be used (Perceptual, Saturation, Relative Colorimetric and Absolute Colorimetric).

Perceptual rendering intent change all the colours of a source space into the colours of a destination space in the way that preserve overall colours relationship. This method is good when converting images that contain significant out-of-gamut colours (Fraser et al., 2005), and it is often used when converting photographs. When rendering colours with Saturation rendering intent, colours are changed so that source space completely fit destination space. Thus it is possible to produce more saturated, vivid colours, and it is mainly used for business and commercial graphics. Colorimetric rendering intents reproduce all colours which are in out-of-gamut colours to the closest reproducible hue. That makes these intents the most precise, because only out-of-gamut colours are changed. However, it also means that more than one colour from source space
$\mathrm{Y}_{\text {minDest }}$ - the minimum $\mathrm{Y}$ value of the destination gamut of destination space exactly the same and clips 
can be mapped into closest single colour of destination space which sometimes result in "cutting off" colours of input device. Relative Colorimetric rendering intent maps white colour of a source to white colour of destination device, while Absolute Colorimetric maintain white colour of input device by changing all the other hues. Figure 2 shows illustration of mapping methods where position of specific colour in presented by dots. Position of a colour before conversion is marked with black, and after mapping with white dot.

It is not necessary to activate BPC option with Perceptual rendering intent, because all the colours of input device (including black) are mapped into the colours of output device. Still, BPC is available for this rendering intent, to be used with malformed profiles. Because of a type of rendering, BPC option is not available for colour conversion using Absolute Colorimetric rendering intent, and it can be activated with Relative Colorimetric and Saturation.

Besides rendering intents, the important role in colour conversions plays colour management module (CMM). Using the information's from profiles, CMM performs all the calculations needed for converting colour from one colour space to another. CMM is usually bound for operating system itself or software manufacturer. Achieving constant colour from one application and device to another on operating system basis without colour management system is extremely hard task. Before ICC, every application used its own specific colour management system, thus complicating the communication between different colour reproduction softwares.
Microsoft, creator of Windows operating system, in Windows 95 implemented its first colour management module - Image Color Management (ICM). ICM version 1 supported ICC system and RGB profiles. Second, improved version of ICM, maintain compatibility with ICC technology with extended list of supported colour spaces (CMYK, Lab), standard colour spaces (sRGB) and also use divers colour management module (as default CMM LinoColor is used, today in Heidelberg's property). This approach enable that user in graphic application (like Photoshop) can decide which CMM to use. Usually, it is ICM or Adobe CMM, which are also tested in this work. With new operating system, Windows Vista, Microsoft presented new colour management system which differs from previous - it has it's own types of profiles, methods for gamut mapping and contains modified CMM with colour transformations that include colour appearance model's equations. The basic concept of Windows Vista system is shown in Figure 3.

Windows Vista also contain upgraded ICM (version 3) which can be used with current ICC technology and ICC profiles. User can decide to use WCS for colour transformations, and it can be activated during hybrid colour management with both ICC and WCS technology. WCS instead present CMM for colour transformations use new CITE system. Beside modified colour calculation, WCS brings in specific profiles based on XML language. Adobe applications do not support WCS directly, but by enabling ICM module and choosing one of the Vista's profiles, colour transformations will be performed through new colour management

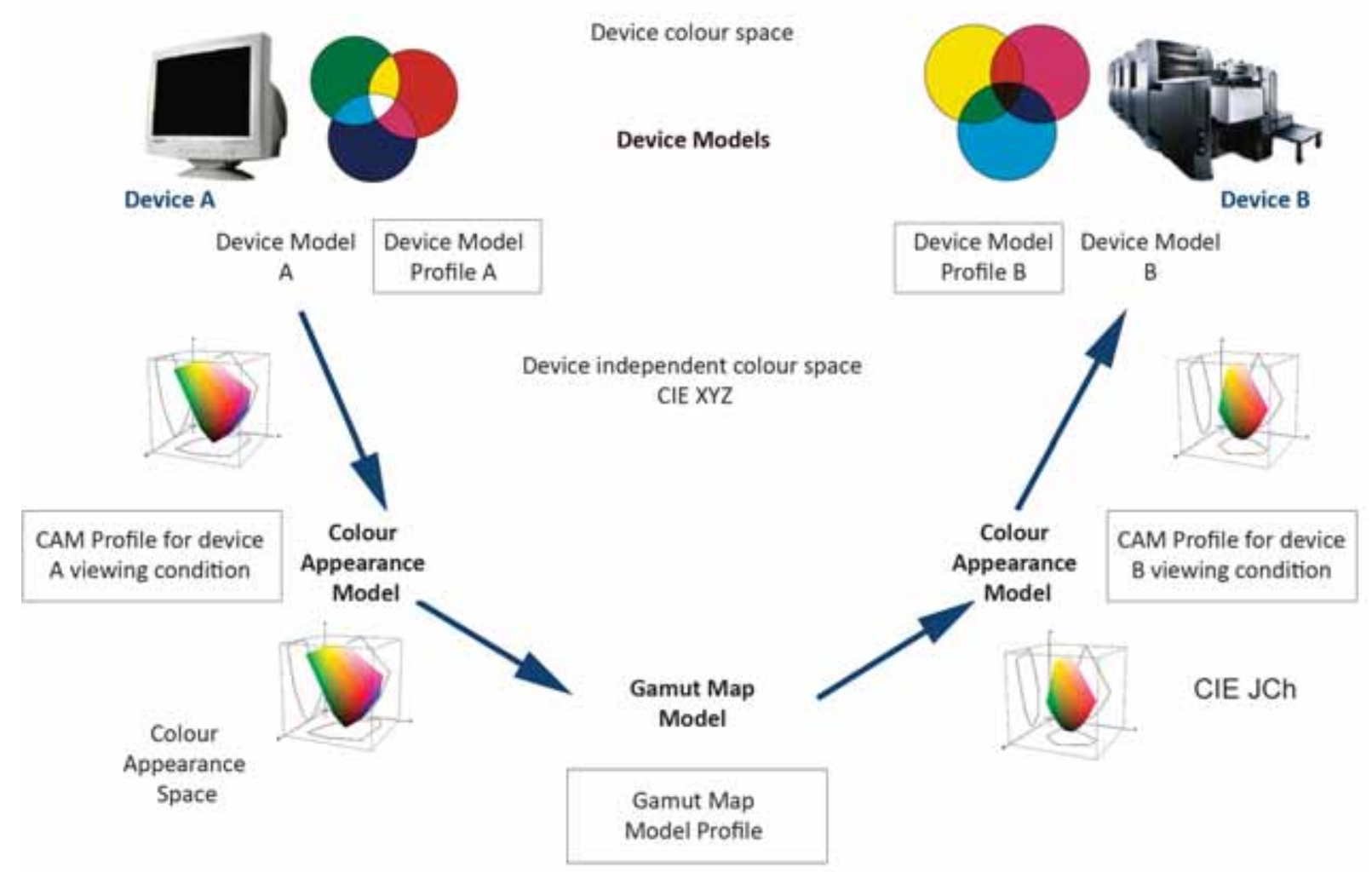

Figure 3. Basic concept of Windows Vista colour management system 
system. Since Vista's WCS calculates the color transformation on the fly and has the exact information for each device's gamut, gamut mapping should be more efficient and accurate. This means that black point compensation is essentially turned on at all times, incorporated into colour transformations, thus producing different results then ICM transformation method.

\section{Method and materials}

In order to define usability of black point compensation while making transformations from one colour space to another, RGB to CMYK conversions of digitally created GretagMacbeth SG test target were performed (due to the fact that conversion is performed from larger to smaller colour space's gamut some clipping of colours must occur). Test target was converted with different rendering intents with or without activating BPC. As a source profile sRGB was chosen (white point values: $\mathrm{L}$ 100, a -2, b -19; black point values: L 0, a 0, b 0), while as destination profile Europe ISO Coated Fogra27, official Fogra standard for printing on coated papers was used (white point values: L 96, a 0, b -3; black point values: L 12, a 0, b -1). As seen in Figure 4, gamut described by SRGB profile is significantly wider than the one of Coated Fogra27.

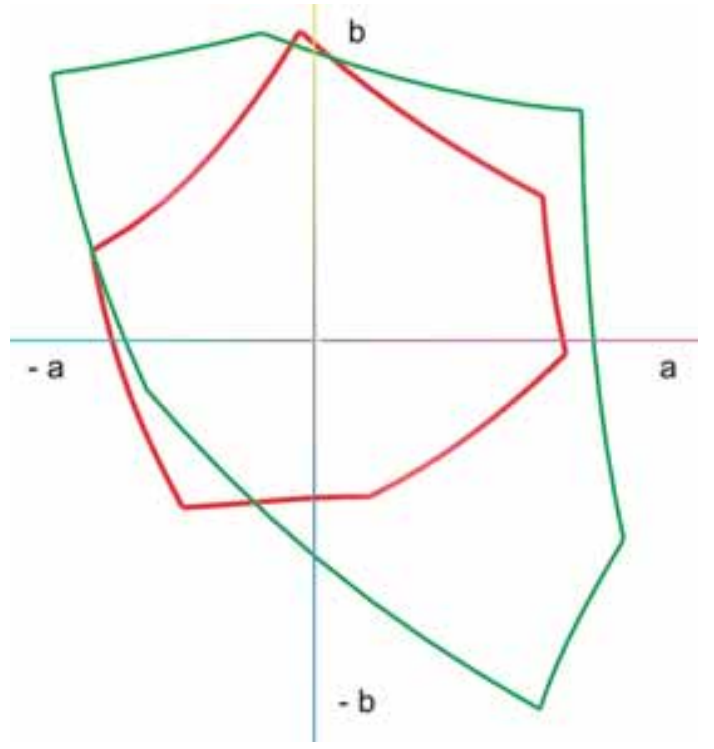

Figure 4. 2D projection of profiles gamuts in Lab colour space

(sRGB gamut marked red, Coated Fogra27 gamut marked green)

Conversions were performed within Adobe Photoshop CS4 where besides rendering intents, colour management modules (ICM and Adobe CMM) were also varied. After conversions allocation of colours on image are observed inside device independent colour space (CIE Lab) within CHROMiX ColorThink software and also in LCH colour space using the Couleur Col-
orSpace software. Changes in hue and lightness after conversion with and without BPC (with certain rendering intent and CMM) are observed. Besides comparing different modules (ICM and ACE) on Windows XP operating system, transformations are also done on Vista OS which use modified modules. WCS colour rendering can be activated if one of the Vista's profiles is used during transformations. Thus, to ensure that WCS is turned on, wsRGB profile instead of sRGB was used as a source space.

In order to estimate whether obtained results correspond to subjective colour perception conversions are also performed for three types of images: high key image (defined as Type 1), middle key image (Type 2) and low key image (Type 3). Images used together with their histograms are shown on Figure 5.

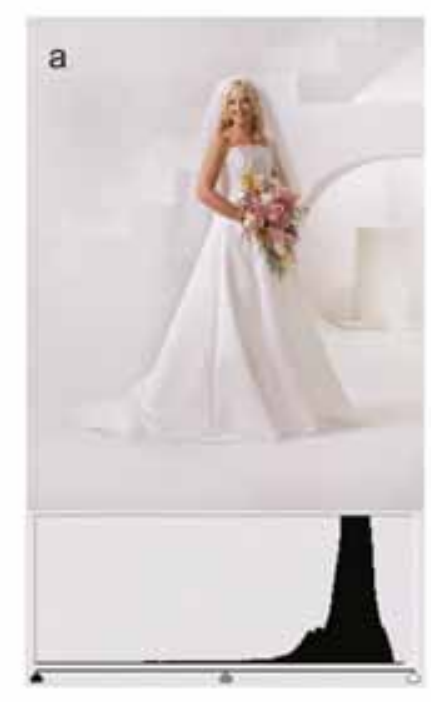

b
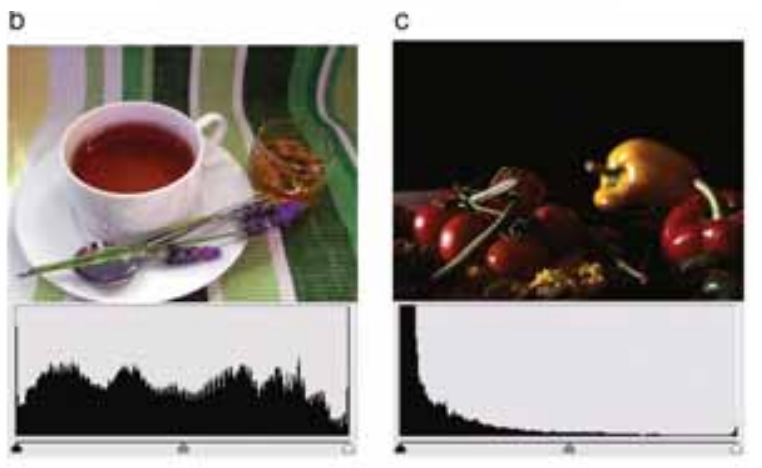

Figure 5. Images used for perceptual evaluations: a. high key image together with corresponding histogram, b. middle key image and its histogram, c. low key image and its histogram.

Basic image and images converted with or without BPC (with certain CMM and rendering intent) are observed in CIE Lab colour space within Photoshop CS4 on calibrated LCD monitor (Samsung T220, calibrated according to ISO 12646:2008). Images are displayed in two rows - basic image in first, and converted in second row. Since surround have a great impact on appearance 
of colours viewed on monitor (Fairchild, 2005) evaluation is performed in dark room so that no flare light can affect colour appearance.

Visual evaluation of converted images regarding to similarity with the basic one was performed. Twenty observers ages from 21-33, all students and employees of department for Graphic engineering and design, took part in visual evaluation. They were all tested for colour blindness with Isihara test and also with Farnsworth Munsell 100 Hue test (within software Color Vision) in order to estimate their ability to distinguish small chroma and luminance variations. The observers were presented with the original image along with pairs of candidate converted images and were asked to pick the most accurate reproduction with respect to the original image. In order to avoid systematic error, due to some persons might prefer one side to another when the images seem indistinguishable (Dugay, 2007), all pairs were presented twice in different order.

\section{Results and discussion}

After test target conversions, allocation of colours in Lab colour space showed that if Perceptual and Saturation rendering intents are used differences in colours position when BPC is and is not activated are negligible. Shifts in hues are not noticed, while with certain colours (purple, green and dark neutrals) small shifts toward higher $\mathrm{L}$ values were detected. This can easily be explained with rendering methods and also by gamuts of profiles used for conversion. Same results are obtained when Microsoft ICM and Adobe (ACE) colour management modules are used.

With Relative Colorimetric rendering intent significant changes in both hue and lightness are noticed if BPC is activated during conversions. Most of the colours are shifted toward higher $\mathrm{L}$ values and $\mathrm{L}$ axis itself (which leads to global saturation reduction). Small differences are noticed if different modules are used. In Figure 6 positions of GretagMacbeth SG test target colours in Lab colour space are shown. When target is converted without BPC activated, colours are presented darker

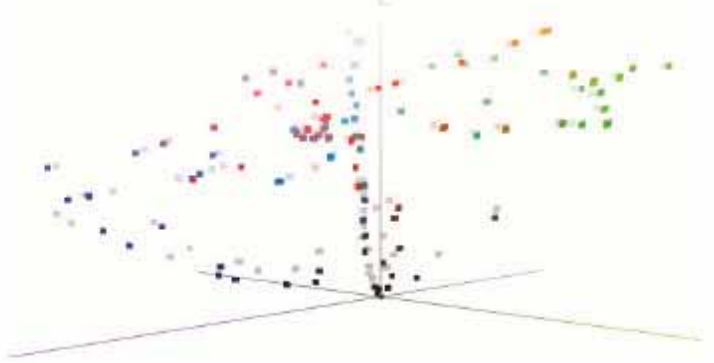

Figure 6. Arrangement of colours from GretagMacbeth SG test target in Lab colour space after converting from RGB to CMY with Relative Colorimetric rendering intent and ACE CMM (when $B P C$ is not activated colours are presented darker) (full density), while when BPC is activated position of colours are marked more transparently.

Black point compensation usually has no effect on neutral light colours. With other colours dislocations are mostly noticed as shifting to higher L values, more expressed with dark tones (Figure 6). These shifts are in accordance with theoretical background of gamut mapping since black point of source profile is on lower $\mathrm{L}$ value. Shifts in hues are observed on 2D gamut projection on $a-b$ axes of Lab colour space (Figure 7). If during conversion from one colour space to another BPC is used, hues are mostly shifted toward L axis, where biggest shifts are noticed at blue, green and also some red tones. These deviations can be explained with the fact that SRGB colour space has wider gamut from chosen CMYK space exactly in mentioned tones (as presented earlier on Figure 4), so these are the most affected during mapping.

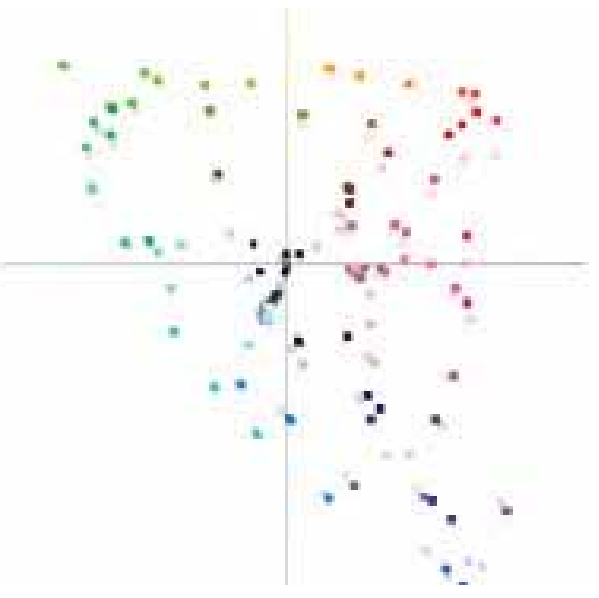

Figure 7. Arrangement of GretagMacbeth SG test target colours after converting from RGB to CMY with Relative Colorimetric rendering intent and ACE CM$2 D$ projection on $a-b$ axes in Lab colour space (when $B P C$ is not activated colours are presented darker)

Same is noticed when hue shifts are observed in LCH colour space (within Couleur Color Space software). Arrangement of colours from test target when converted with or without BPC activated in LCH colour space are presented in Figure 8.

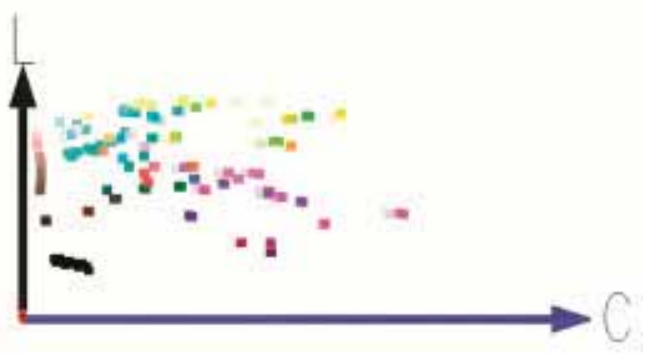

Figure 8. Arrangement of GretagMacbeth SG test target colours as a $2 D$ projection of gamuts on $L-C$ axes of $L C H$ colour space after converting from RGB to CMY with Relative Colorimetric rendering intent and ACE CMM (when BPC is not activated colours are presented darker) 
Colour shifts when ICM module and Relative Colorimetric rendering are used for conversion are very similar to previous. Colours also shifted toward higher L values, and dark neutral tones are, again, mostly affected. The only noticeable difference is that purple tones are less shifted when ICM module is used, which can be seen on Image 9 where projection to a-b axes of $\mathrm{Lab}$ colour space is presented.

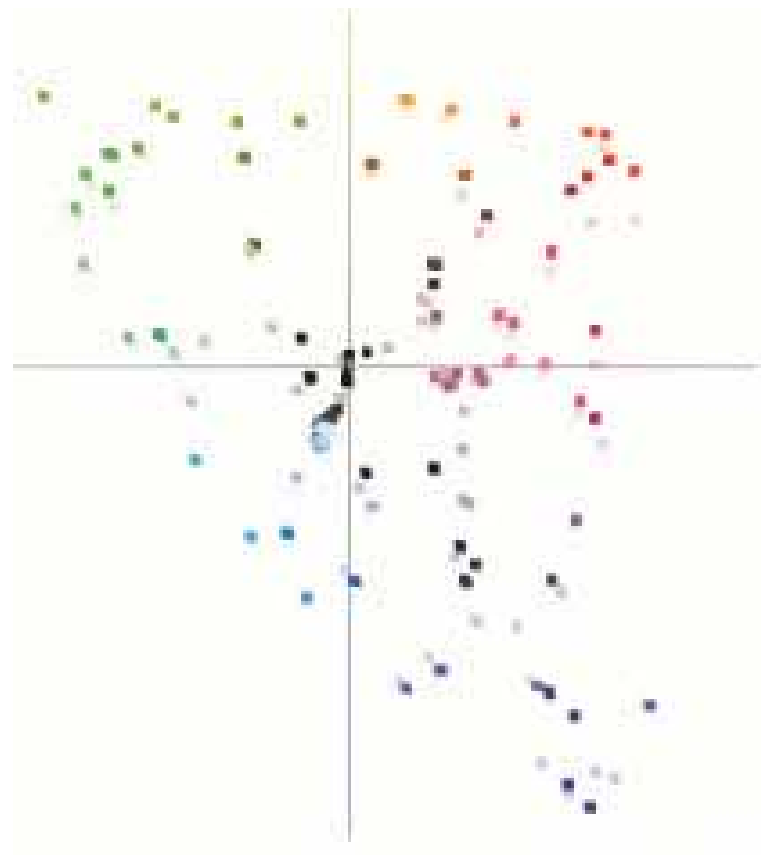

Figure 9. Arrangement of GretagMacbeth SG test target colours after converting from RGB to CMY with Relative Colorimetric rendering intent and ICM CM $2 D$ projection on $a-b$ axes in Lab colour space (when BPC is not activated colours are presented darker)
Results obtained by visual assessment of converted images are presented in Table 1 and 2. Here, the preferred images (with respect to the original), which most of the observers chose, are marked with plus sign. It means that image converted with given rendering intent is regarded as better reproduction of original image. Sign is not placed if observes couldn't distinguish difference in displayed images. In the case where rendering with ACE module is assessed, 16 observers (80\%) gives the answers presented in Table 1 , while with the ICM module with Perceptual and Saturation rendering only 2 observers gave different answers from the others i.e. noticed difference in rendering with and without BPC. Since it is only $10 \%$ of all the observers and both judgments were different, those are not regarded as valid results.

With Perceptual and Saturation rendering observers usually couldn't perceive any difference in displayed images, which confirmed conclusions deducted by observing colours shifts in Lab colour space. When Relative Colorimetric rendering is used better results are obtained with activated BPC, since details in dark areas are preserved. Exception is rendering high key images where better reproduction of original image is gained without BPC (was noticed when ICM module is used). This can be explained by colours of the image itself. In this case, using BPC result in all tones shifts, and since there are very few dark tones in image which is to be mapped result is incorrect reproduction. These deviations are not large, but if precise reproduction is goal, they should be taken into account.

Second part of testing included estimation of the differences in rendering on Windows Vista OS. ACE module (with activated BPC) and Vista's WCS colour manage-

Table 1. Results of visual assesment when using ACE module on Windows XP

\begin{tabular}{|c|c|c|c|c|c|c|}
\hline \multirow{2}{*}{$\begin{array}{c}\text { Type of the } \\
\text { image used }\end{array}$} & \multicolumn{2}{|c|}{$\begin{array}{c}\text { Relative Colorimetric } \\
\text { rendering }\end{array}$} & \multicolumn{2}{c|}{ Perceptual rendering } & \multicolumn{2}{c|}{ Saturation rendering } \\
\cline { 2 - 7 } & Without BPC & $\begin{array}{c}\text { With } \\
\text { BPC }\end{array}$ & Without BPC & $\begin{array}{c}\text { With } \\
\text { BPC }\end{array}$ & Without BPC & $\begin{array}{c}\text { With } \\
\text { BPC }\end{array}$ \\
\hline Type 1 & & & + & & + & \\
\hline Type 2 & & + & & & & \\
\hline Type 3 & & + & & & & \\
\hline
\end{tabular}

Table 2. Results of visual assesment when using ICM module on Windows XP

\begin{tabular}{|c|c|c|c|c|c|c|}
\hline \multirow{2}{*}{$\begin{array}{c}\text { Type of the } \\
\text { image used }\end{array}$} & \multicolumn{2}{|c|}{$\begin{array}{c}\text { Relative Colorimetric } \\
\text { rendering }\end{array}$} & \multicolumn{2}{c|}{ Perceptual rendering } & \multicolumn{2}{c|}{ Saturation rendering } \\
\cline { 2 - 7 } & Without BPC & $\begin{array}{r}\text { With } \\
\text { BPC }\end{array}$ & Without BPC & $\begin{array}{r}\text { With } \\
\text { BPC }\end{array}$ & Without BPC & $\begin{array}{r}\text { With } \\
\text { BPC }\end{array}$ \\
\hline Type 1 & + & & & & & \\
\hline Type 2 & & + & & & & \\
\hline Type 3 & & + & & & & \\
\hline
\end{tabular}


ment system, in which BPC is always activated are taken into consideration. Here, as mentioned, wcsRGB was chosen, in order to ensure that WCS rendering is used. In Figure 10 colours of GretagMacbeth SG test target after conversion with both rendering methods and Relative Colorimetric intent are shown as a projection on $\mathrm{a}-\mathrm{b}$ axes in Lab colour space. It can be noticed that saturation achieved with Adobe CMM (ACE) is somewhat lower then the one gained when WCS is applied. The same is noticed when Perceptual and Saturation rendering intent are used. Changes in saturation level are very small, and thus it can be concluded that ACE CMM with activated BPC and WCS (where mapping is always performed with compensating black levels) gives pretty much the same colours rendering results.

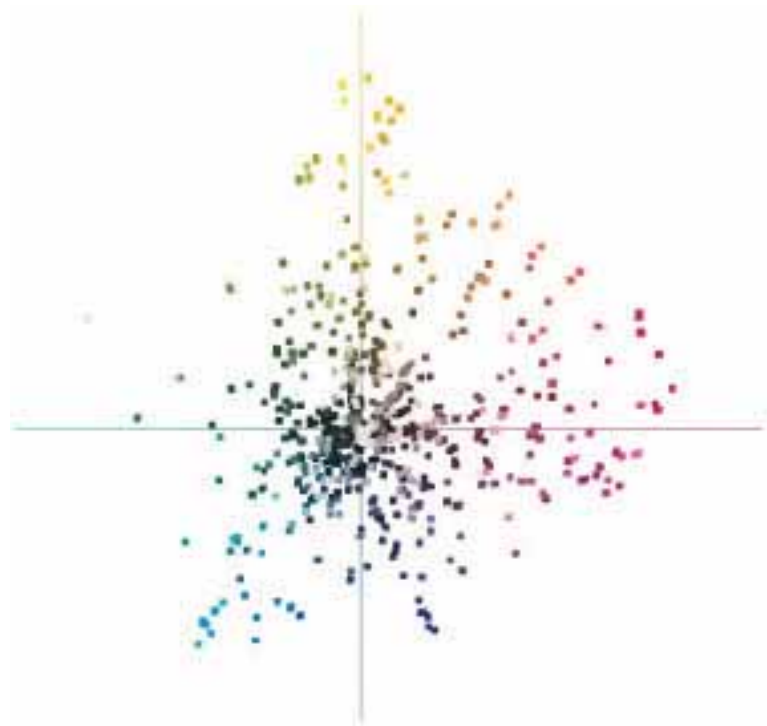

Figure 10. Arrangement of GretagMacbeth SG test target colours after converting from RGB to CMY with Relative Colorimetric rendering intent on Windows Vista OS - 2D projection on a-b axes in Lab colour space (when ACE module is used for transformations colours are presented darker, when WCS CMM is used colours are shown more transparently)

\section{Conclusion}

Based on the presented results it can be concluded that black point compensation is not effective for every types of images. Usability depends more of desired rendering intent, and less of chosen CMM. Difference in colour appearance is noticeable when conversions are performed with Relative Colorimetric rendering, while with other rendering intents effect of black point compensation is not emphasized regardless of the CMM used. Neutral dark tones are the most affected with BPC and also the tones which our out of destination space's gamut. Thus, BPC is the most useful when converting low key images and with images where some details in dark areas have to be preserved. Activating BPC with high key images leads to inaccurate reproduction, and therefore in cases when high key images are to be converted BPC should not be taken into consideration. With all other image types better results are obtained if BPC is turned on during conversion. When performing transformations on Windows Vista OS pretty much the same results are obtained with ACE CMM where BPC is turned on and Vista's native CMM.

\section{References}

1. Adobe Systems Incorporated. (2006) Adobe Systems' Implementation of Black Point Compensation. [Online] Avaliable from: http://www.color. org/AdobeBPC.pdf [Accessed 5th February 2009].

2. Bonnier, N., Schmitt, F., Leynadier, C. (2008) Improvement in Spatial Gamut Mapping Algorithms. In: Society for Imaging Science and Technology. Proceedings of 4th European Conference on Colour in Graphics, Imaging and Vision, 10 - 13 June 2008, Terrassa, Spain, pp. 341-346.

3. Dugay, F., Ivar F., Hardeberg J. (2007). Perceptual Evaluation of Gamut Mapping Algorithms, Color Research and Application [Online] 26 (1), 470-476. Avaliable from: http://www.ansatt.hig. no/ivarf/publications/Dugay_08_cra.pdf [Accessed 5th February 2009].

4. Fraser, B., Murphy, C., Bunting,F. (2005). Real World Color Management. 2nd Ed. Berkeley, Peachpit Press.

5. Morovič, J. (2008). Color Gamut Mapping. Chichester, John Wiley and Sons, Ltd

6. Nate, J. (2004). Black point compensation-Thumbs up or down?. Newspapers and Technology Magazine. [Online] Avaliable from: http://www.newsandtech.com/issues/2004/05-04/pt/05-04_blackpoint.htm [Accessed 6th February 2009].

7. Fairchild, M. (2005). Color Appearance Models. 2nd Ed. Chichester, John Wiley and Sons, Ltd. 